007

MULTI-MODAL IMAGING OF ANOMALOUS LEFT CORONARY ARTERY OFF THE PULMONARY ARTERY (ALCAPA)

${ }^{1}$ Kavin Jayawardhana, ${ }^{2}$ Ausami Abbas, ${ }^{2}$ Stephen Harden, ${ }^{2}$ Charles Peebles, ${ }^{2}$ James Shambrook. 'Queen Alexandra Hospital, Portsmouth, UK; ${ }^{2}$ Southampton General Hospital, Southampton, UK

\subsection{6/heartjnl-2016-309680.7}

Introduction Although first described in 1866, ALCAPA was recognised with a clinical description and correlation with autopsy findings by Bland and colleagues in 1933. ALCAPA accounts for approximately $0.25-0.5 \%$ of all congenital heart defects and exists predominantly in two forms; infant type and adult type. Infants present with features of myocardial ischaemia and heart failure and very rarely can be associated with other cardiac anomalies. Mortality rate approaches $90 \%$ in infants if ALCAPA is left untreated. Adult cases are unusual and but can present with unusual ischaemic symptoms. Although historically ALCAPA was diagnosed at conventional angiography, the development of gated CT and MRI imaging allows assessment with non-invasive means. Learning Objectives: To describe and illustrate radiological findings and characteristics in patients diagnosed with having an ALCAPA anomaly.

Imaging Findings We describe our case series of patients with characteristic findings on electrocardiography, CT and MRI seen in patients with ALCAPA. The left coronary artery arises from the pulmonary trunk and branches into dilated LAD and circumflex vessels. Collaterals are usually seen between the right and left coronary arteries with retrograde flow through the left coronary artery into the pulmonary trunk. The right coronary artery is often also dilated due to high volume flow. Identification of these dilated collaterals is often the first indication of this pathology.

Conclusion It is important for radiologists to be aware of the typical imaging findings in this relatively rare anomaly as prognosis is good with early diagnosis and surgical correction.

\section{COMPARISON OF WEIGHT-BASED VS ESTIMATED CONTRAST DOSE TECHNIQUES IN CORONARY COMPUTED TOMOGRAPHY ANGIOGRAPHY}

Will Loughborough, Nathan Manghat, Mark Hamilton. Bristol Royal Infirmary, University Hospitals NHS Trust, Bristol, UK

\subsection{6/heartjnl-2016-309680.8}

Introduction The purpose of this study was to compare weight based versus estimated contrast dosing techniques in coronary computed tomography angiography (CCTA).

Methods This single centre retrospective observational study compared 47 patients undergoing CCTA. All imaging was performed on a 320 slice scanner at $100 \mathrm{kV}$ with bolus tracking. In the weight based protocol, patients received $22 \mathrm{mg} / \mathrm{kg} / \mathrm{s}$ of contrast for $14 \mathrm{~s}$. In the estimated group, contrast dosing and rate was estimated by a consultant cardiac radiologist. Two tailed t tests determined significance between patient and contrast variables. Enhancement of cardiac chambers and coronary arteries was calculated through region of interest areas in Hounsfield units (HUs).

Results There were no significant differences between the groups in terms of mean weight $(\mathrm{p}=0.42)$ or mean heart rate $(\mathrm{p}=0.29)$. The estimated group received a significantly higher volume of contrast than the weight based group $(71 \mathrm{mls}$ vs $58 \mathrm{mls}, \mathrm{p}=<0.001)$. There was a non-significant difference between groups in MEV of coronaries. Both groups produced diagnostic MEVs above 400 HUs. Right sided chambers were attenuated significantly more in the best guess than the weight based group, for example right ventricle MEV 279 vs 141 ( $\mathrm{p}=$ 0.01). SDs in coronary arteries were similar between groups but weight based contrast protocol achieved lower standard deviation of HUs on the right sided chambers.

Conclusion Weight based and estimated contrast regimes produce similar variability and adequate opacification in coronary arteries in CCTA. However, weight adjusted contrast dosing technique administers a lower dose of contrast, with lower enhancement of the right sided chambers.

\section{INCIDENTAL FINDINGS IN CTCA}

Julie Morrison, Liz Philip. Golden Jubilee National Hospital, Glasgow

\subsection{6/heartjnl-2016-309680.9}

Background CT coronary angiograms have been performed at the Golden Jubilee National Hospital since 2004 and with the installation of the GE 750 HD scanner in 2010, a cardiologist led service for CTCA was set up. To comply with BSCI guidelines, the non-contrast calcium score was reconstructed to provide a larger field of view for radiologists to report non-cardiac elements of the scans. After discussion with the lead radiologist, a decision was made to split both non-contrast and contrast series and retrospectively reconstruct them into larger display field of view to allow radiologists to report the non-cardiac elements from both series. This audit was carried out to investigate the number of incidental radiological findings reported on CTCA scan and their clinical significance.

Method Data was collected retrospectively from the non-cardiac section of reports generated from CTCA scans performed between February-July 2015 inclusive. Any incidental findings were noted and recorded.

Results 407 CTCA scans were performed between February-July 2015, within this group of 173 patient scans, 42\%, were reported with incidental findings. Most significantly within this cohort, 4 patients (2\%) required immediate treatment for pulmonary embolus. 2 patients $(1 \%)$ required urgent referral for suspicious chest mass. 2 patients (1\%) required 3-4 month CT follow up for chest mass.

Conclusion A large proportion of patients scanned were reported with incidental findings in the chest/upper abdominal area. The majority were benign, requiring no urgent follow up. However a small percentage was reported to have significant clinical findings which required urgent or immediate referral/ treatment. Therefore the audit has demonstrated the importance of reconstructing both pre and post contrast large field of view images in order to visualise pathology which may be hard to distinguish in the non contrast phase. The audit has also highlighted the importance of a multi-disciplinary approach when reporting both cardiac and non-cardiac elements of the CTCA scan. 